\title{
Article \\ Comparing the Fasting and Random-Fed Metabolome Response to an Oral Glucose Tolerance Test in Children and Adolescents: Implications of Sex, Obesity, and Insulin Resistance
}

\author{
Jennifer L. LaBarre ${ }^{1,2, *}$, Emily Hirschfeld ${ }^{3}$, Tanu Soni ${ }^{4}$, Maureen Kachman ${ }^{4}$, Janis Wigginton ${ }^{4}$, \\ William Duren ${ }^{4,5}$, Johanna Y. Fleischman ${ }^{6}$, Alla Karnovsky ${ }^{5}$ (D), Charles F. Burant ${ }^{1,6,+}$ and Joyce M. Lee ${ }^{3,7, *,+}$
}

check for updates

Citation: LaBarre, J.L.; Hirschfeld, E. Soni, T.; Kachman, M.; Wigginton, J.; Duren, W.; Fleischman, J.Y.; Karnovsky, A.; Burant, C.F.; Lee, J.M. Comparing the Fasting and Random-Fed Metabolome Response to an Oral Glucose Tolerance Test in Children and Adolescents: Implications of Sex, Obesity, and Insulin Resistance. Nutrients 2021, 13, 3365. https://doi.org/10.3390/ nu13103365

Academic Editor: Francis Finucane

Received: 2 July 2021

Accepted: 23 September 2021

Published: 25 September 2021

Publisher's Note: MDPI stays neutral with regard to jurisdictional claims in published maps and institutional affiliations.

Copyright: (C) 2021 by the authors Licensee MDPI, Basel, Switzerland. This article is an open access article distributed under the terms and conditions of the Creative Commons Attribution (CC BY) license (https:/ / creativecommons.org/licenses/by/ $4.0 /)$.
1 Department of Internal Medicine, University of Michigan Medical School, Ann Arbor, MI 48109, USA; burantc@med.umich.edu

2 Department of Medicine, Dartmouth-Hitchcock Medical Center, Weight and Wellness Center, Lebanon, NH 03766, USA

3 Susan B Meister Child Health Evaluation and Research Center, Department of Pediatrics, University of Michigan, Ann Arbor, MI 48109, USA; ehirschf@med.umich.edu

4 Michigan Regional Comprehensive Metabolomics Resource Core, University of Michigan, Ann Arbor, MI 48109, USA; tanusoni@umich.edu (T.S.); mkachman@umich.edu (M.K.); wiggie@med.umich.edu (J.W.); wld@med.umich.edu (W.D.)

5 Department of Computational Medicine and Bioinformatics, University of Michigan Medical School, Ann Arbor, MI 48109, USA; akarnovs@med.umich.edu

6 Department of Molecular and Integrative Physiology, University of Michigan, Ann Arbor, MI 48109, USA; johf@umich.edu

7 Division of Pediatric Endocrinology, Department of Pediatrics, University of Michigan, Ann Arbor, MI 48109, USA

* Correspondence: jennifer.l.labarre@hitchcock.org (J.L.L.); joyclee@med.umich.edu (J.M.L.)

+ These authors contributed equally to this work.

Abstract: As the incidence of obesity and type 2 diabetes (T2D) is occurring at a younger age, studying adolescent nutrient metabolism can provide insights on the development of T2D. Metabolic challenges, including an oral glucose tolerance test (OGTT) can assess the effects of perturbations in nutrient metabolism. Here, we present alterations in the global metabolome in response to an OGTT, classifying the influence of obesity and insulin resistance (IR) in adolescents that arrived at the clinic fasted and in a random-fed state. Participants were recruited as lean $(n=55$, aged $8-17$ years, BMI percentile $5-85 \%$ ) and overweight and obese (OVOB, $n=228$, aged $8-17$ years, BMI percentile $\geq 85 \%$ ). Untargeted metabolomics profiled 246 annotated metabolites in plasma at $\mathrm{t} 0$ and $\mathrm{t} 60$ min during the OGTT. Our results suggest that obesity and IR influence the switch from fatty acid (FA) to glucose oxidation in response to the OGTT. Obesity was associated with a blunted decline of acylcarnitines and fatty acid oxidation intermediates. In females, metabolites from the Fasted and Random-Fed OGTT were associated with HOMA-IR, including diacylglycerols, leucine/isoleucine, acylcarnitines, and phosphocholines. Our results indicate that at an early age, obesity and IR may influence the metabolome dynamics in response to a glucose challenge.

Keywords: oral glucose tolerance test; metabolomics; fatty acids; adolescents; acylcarnitines; obesity; insulin resistance; glucose challenge

\section{Introduction}

As the prevalence of prediabetes and type 2 diabetes (T2D) in adolescents is increasing [1], it is vital to identify metabolic dysfunction prior to disease onset to classify individual risk and implement preventative strategies. Classically, an oral glucose tolerance test (OGTT) diagnoses impaired glucose tolerance (IGT)/prediabetes and T2D, by measuring the acute trajectory of glucose in response to ingestion a 75-g glucose solution. Profiling the metabolome in response to an OGTT can provide a deeper phenotyping of T2D risk, 
expanding upon measuring traditional glucose levels to a profile of metabolic response across multiple pathways. Several studies have demonstrated the widespread response of the metabolome to an OGTT in adults, observing alterations in proteolysis, lipolysis, ketogenesis, and glycolysis in healthy individuals in response to the challenge [2,3]. These studies suggest an acute increase of glycolytic intermediates and rapid inhibition of lipolysis [4] and proteolysis [5], as evidenced by decreases in amino acids, free fatty acids (FFA), and acylcarnitine (AC) intermediates of beta-oxidation.

Adolescent obesity is a strong risk factor for the development of T2D, as a nationwide study observed that severe obesity increases the incidence of T2D in early adulthood in both males and females [6]. In fasted plasma, obesity is associated with alterations in the metabolome, including elevations in lipids, branched chain amino acids (BCAA), and aromatic amino acids [7-9]. In adults, the metabolome response to an OGTT was profiled in the context of obesity, observing a delayed reduction of FFA and higher levels of amino acids, including isoleucine and leucine, at $30 \mathrm{~min}$ post-OGTT in adults with obesity [10]. In adolescents, Müllner et al. [11] identified metabolites in response to an OGTT associated with obesity, including elevations of AC 2:0, glutamate, alanine, and pyruvate, suggesting a mismatch between beta-oxidation and TCA-cycle activity.

Pediatricians are more likely to order non-fasting tests compared with a gold standard fasting OGTT because of the inconvenience of fasting tests [12]. As a result, a random plasma glucose sample and a 1-h non-fasting glucose challenge have been assessed for the prediction of T2D, offering strong discrimination for identifying prediabetes in adolescents [13]. Furthermore, evidence has suggested a tight link between post-meal glucose levels and T2D complications [14], supporting the utility of a random-fed glucose challenge. Although the fasted and non-fasted metabolome have established differences [15], it is uncertain how the metabolome response to an OGTT differs in individuals arriving to the clinic fasted and in a random-fed state. In addition, the influence of insulin resistance (IR) on the fasted vs. random-fed metabolome response to an OGTT is unknown. At fasting, multiple metabolic pathways are associated with IR and T2D including BCAA metabolism [16], beta oxidation [17], and bile acids [18]. In adults arriving to the clinic fasted, Nowak et al. observed correlations between AC response to the OGTT with a degree of IR, as individuals with worsening IR had a blunted decline of medium-chain ACs, products of beta-oxidation [19].

Our main objectives were to assess the metabolome response to an OGTT by (1) comparing the response to a fasted OGTT in participants with overweight and obesity with that in controls and (2) determining differential metabolite responses to a fasted vs. random-fed OGTT among participants who were overweight and had obesity. Furthermore, we sought to identify metabolite responses to an OGTT associated with insulin resistance (as measured by HOMA-IR) in participants who were overweight and had obesity at the Fasted and Random-Fed Visit, considering the influence of sex. Results from these analyses identified a panel of metabolites that can be profiled by fasted or random glucose challenges with the potential to predict longitudinal T2D risk.

\section{Materials and Methods}

\subsection{Research Design}

The cohort consisted of adolescents who were either overweight or obese (OVOB) (BMI percentile $\geq 85$ th for sex/age [20]) and lean adolescents (BMI percentile $<85$ th for sex/age [20]), aged 8-17 years at time of enrollment, recruited from primary care and pediatric specialty clinics in southeast Michigan (2015-2018). Individuals were excluded if they had known diabetes, use of medications known to affect glucose metabolism (oral steroids, metformin, insulin, or sulfonylureas), verbal report of pregnancy, or acute or chronic infections. Written informed consent was obtained from the parent/guardian for all participants and participants $\geq 10$ years provided written assent. The study was approved by the University of Michigan Institutional Review Board. 
Participants attended study visits at the Michigan Clinical Research Unit, where a medical history, vital signs, anthropometrics, and laboratory evaluation were performed. During the Fasted OGTT Challenge, OVOB $(n=228)$ and lean participants $(n=55)$ arrived after an overnight fast for a formal OGTT (Figure 1), with fasting times ranging from $9 \mathrm{~h}$ and $35 \mathrm{~min}$ to $19 \mathrm{~h}$ and $21 \mathrm{~min}$ (Figure S1a). The OGTT dosage consisted of $1.75 \mathrm{~g}$ glucose $/ \mathrm{kg}$ body weight, with the maximum dosage of $75 \mathrm{~g}$ glucose (Glucola, Fisherbrand) (Figure S1b). Blood samples were drawn at baseline (t0) and every $30 \mathrm{~min}(\mathrm{t} 30, \mathrm{t} 60$, t90, and t120 $\mathrm{min}$ ) following the challenge. Glucose and insulin levels were profiled from blood samples collected at $\mathrm{t} 0, \mathrm{t} 30, \mathrm{t} 60, \mathrm{t} 90$, and $\mathrm{t} 120$ min following the OGTT. The untargeted metabolome was profiled from blood samples collected at t 0 and $160 \mathrm{~min}$ following the fasted OGTT. Approximately a week later, OVOB individuals $(n=228)$ returned for a glucose challenge in a random fed state, where participants were not given instructions on the timing of their last meal (50-g, Random-Fed OGTT challenge) (Figure 1), with fasting times varying from $5 \mathrm{~min}$ to $14 \mathrm{~h}$ and $16 \mathrm{~min}$ ( $n=166$ reported last mealtimes) (Figure S1c). Our objective was to mimic a random OGTT that is already performed in the clinic as a screening test for gestational diabetes. The 50-g random OGTT has been previously shown have reasonable discrimination for identifying children with prediabetes [13]. Glucose and insulin levels were profiled from blood samples collected at $\mathrm{t} 0$ and $\mathrm{t} 60 \mathrm{~min}$ following the OGTT. The untargeted metabolome was profiled from blood samples collected at $\mathrm{t} 0$ and t60 min following the Random-Fed OGTT.

Fasted OGTT Challenge

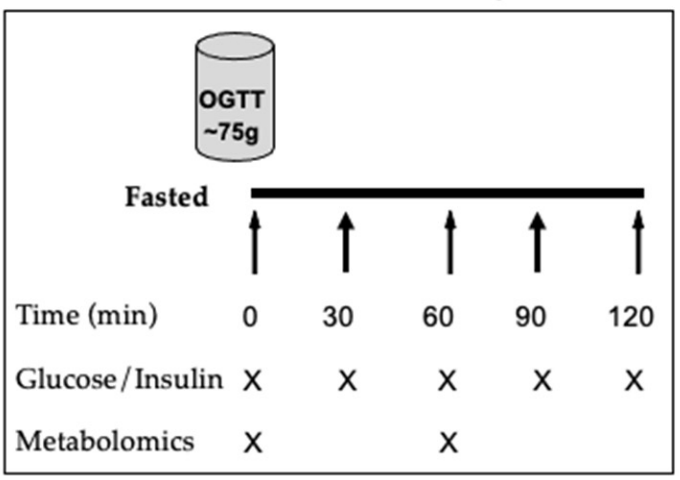

Random-Fed OGTT Challenge

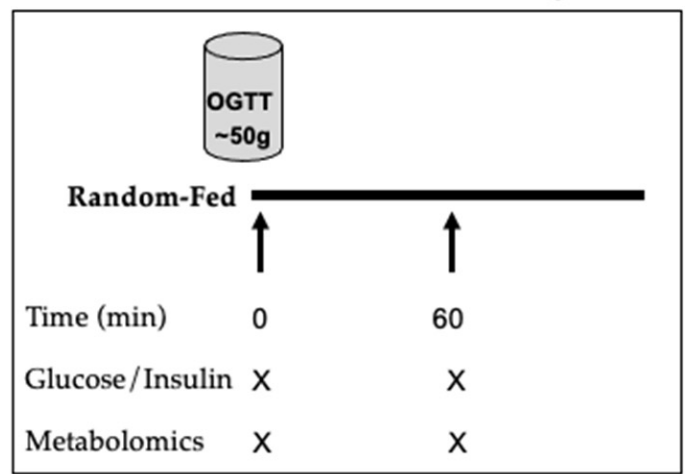

\begin{tabular}{|c|c|c|}
\hline & Fasted Visit & Random-Fed Visit \\
\hline Age (years) & $12.9(8-17)$ & $12.9(8-18)$ \\
\hline OVOB $(\mathrm{n})$ & 228 & 228 \\
\hline lean $(\mathrm{n})$ & 55 & 0 \\
\hline
\end{tabular}

Figure 1. Study Design. Overweight and obese (OVOB) and lean participants were recruited prior to the first visit (Fasted Visit), where an oral glucose tolerance test (OGTT) $(75 \mathrm{~g})$ was administered in the fasted state. The OVOB participants returned approximately a week later for an OGTT (50 g) in a random fed state (Random-Fed Visit). Blood samples were collected before and during the OGTT and used for glucose, insulin, and untargeted metabolomics assays. Mean age and range of ages reported (years).

\subsection{Laboratory Measurements}

The Michigan Diabetes Research Center (MDRC, Ann Arbor, USA) laboratory performed glucose homeostasis assays. Glucose was measured using the glucose hexokinase method and run on a Randox rX Daytona chemistry analyzer (Randox Laboratories Limited, Crumlin, UK). Insulin was profiled using a double-antibody radioimmunoassay [21]. The homeostatic model assessment for insulin resistance (HOMA-IR) utilized glucose and insulin measurement to estimate insulin resistance and beta cell function [22]. Glucose area 
under the curve (AUC) was estimated between $\mathrm{t} 0$ and $\mathrm{t} 120 \mathrm{~min}$ by integrating, using the trapezoid method and stopping when glucose values dropped below baseline (GraphPad PrismVersion 8.4.3). Insulin AUC was estimated between $\mathrm{t} 0$ and $\mathrm{t} 120$ by integrating using the trapezoid method (GraphPad PrismVersion 8.4.3). Hemoglobin A1c (HbA1c) was determined using a Tosoh G7 HPLC Analyzer (Tosoh Biosciences Inc., San Francisco, CA, USA). Biologically implausible outliers were removed ( $n=1$ removed from $t 0$ insulin value). Impaired fasting glucose (IFG) was defined as fasting plasma glucose $\geq 100 \mathrm{mg} / \mathrm{dL}$; impaired glucose tolerance (IGT) was defined as the 2 -h glucose $\geq 140 \mathrm{mg} / \mathrm{dL}$; and prediabetes was defined as IFG, IGT, or HbA1c between 5.7-6.4\% [23].

\subsection{Untargeted Metabolomics}

Untargeted metabolomics analyses were performed by the Michigan Regional Comprehensive Metabolomics Resource Core (MRC ${ }^{2}$ ) (Ann Arbor, MI, USA). Metabolites were extracted from plasma samples using a solvent of methanol, acetonitrile, and acetone (1:1:1) including internal standards (100 mL extraction solvent and $4 \mathrm{~mL}$ internal standards). Samples were reconstituted with a solvent containing methanol and $\mathrm{H}_{2} \mathrm{O}$ (2:98). Untargeted metabolomics was performed on an Agilent system consisting of an Infinity Lab II UPLC coupled with a 6545 qTOF mass spectrometer (Agilent Technologies, Santa Clara, CA) using a JetStream electrospray ionization source. The eluent was analyzed in both positive and negative ion mode electrospray ionization. Chromatographic peaks, representative of metabolite features, were detected using a modified version of existing commercial software (Agilent MassHunter Qualitative Analysis). Data normalization accounted for drift removal within and between batches by utilizing pooled reference samples that were analyzed within each batch using the Systematic Error Removal using Random Forest (SERRF) method. Metabolites were identified via comparing their MS/MS spectra to authentic standards, purchased internal or external standards ran on the same instrument. For this analysis, annotated metabolites $(n=246)$ were selected. Missing peak intensities were imputed by $\mathrm{K}$-nearest neighbor $(\mathrm{K}=5)$ in metabolites with $\geq 70 \%$ detection across samples. R package "impute" was used for imputation. Metabolites with less than 70\% detection across samples were removed. Biologically implausible metabolite peak intensity values were removed.

\subsection{Statistical Analyses}

Descriptive statistics were computed for categorical variables (Pearson's chi-square test) and continuous variables (unpaired Students' $t$-test), stratified by OVOB and lean. Sex-stratified analyses were evaluated. Main analysis objectives are outlined in Figure S2. Peak intensities (PI) of metabolites were utilized for statistical analyses.

Metabolite differences were identified between OVOB and lean at the Fasted Visit (Figure S2a). At t0, linear regression models were run assessing the effect of group ( $\beta_{\text {group }}$, OVOB or lean) on metabolite levels (log2 and standardized), adjusting for sex and age at the Fasted Visit.

$$
\text { Metabolite }=\beta_{\mathrm{o}}+\beta_{\text {group }} X+\beta_{\text {age }} X+\beta_{\text {sex }} X+\epsilon_{i}
$$

Differential metabolites were identified using an adjusted $p$-value (false discovery rate $[F D R]<0.1)[24]$. Positive $\beta_{\text {group }}$ values represent elevations in OVOB and negative $\beta_{\text {group }}$ values represent lower in OVOB. Differential metabolites were selected for metabolite set enrichment analysis (MSEA) [25] to identify biologically meaningful pathways associated with BMI in the metabolomics data. Human Metabolome Database (HMDB) IDs were mapped to 58 of the 66 differential metabolites at $\mathrm{t} 0$. Pathway enrichment analysis used the Small Molecular Pathway Database (SMPDB), which includes 99 metabolite sets based on normal human metabolic pathways. Over Representation Analyses (ORA) with the hypergeometric test was used to determine if metabolite pathways are represented more than expected by chance, denoting significance using a one-tailed $p$-value (unadjusted and FDR reported). Enrichment analyses were run through Metaboanalyst 4.0 [26]. At t0, sex 
differences in the metabolome were considered using unpaired Student's $t$-test (Figure S2a). Differential metabolites were identified using an adjusted $p$-value (FDR $<0.05)$.

Paired $t$-tests distinguished metabolites that significantly differed between $\mathrm{t} 0$ and t60 in each group and state (lean-Fasted, OVOB-Fasted, and OVOB-Random-Fed) using untransformed PI (FDR < 0.05) (Figure S2b). Fold changes were calculated to represent metabolite response using $\log 2(\mathrm{t} 60 \mathrm{PI} / \mathrm{t} 0 \mathrm{PI})$. To assess if the metabolite response to the OGTT was associated with BMI group, unpaired $t$-tests distinguished variations in metabolite fold changes between OVOB and lean at the Fasted Visit (FDR < 0.1) (Figure S2c).

OVOB participants returned to the clinic for a Random-Fasted OGTT Challenge. Differences in glucose and insulin levels between the Fasted and Random-Fed OGTT Challenge were computed (paired $t$-test). Linear regression models were run separately at $t 0$ and t60 assessing the influence of state ( $\beta_{\text {state, }}$, Fasted or Random-Fed) on metabolite levels ( $\log 2$ and standardized across state at each time), adjusting for sex and age at the Fasted Visit (Figure S2d),

$$
\text { Metabolite }=\beta_{\mathrm{o}}+\beta_{\text {state }} X+\beta_{\text {age }} X+\beta_{\text {sex }} X+\epsilon_{i}
$$

Differential metabolites were identified using an adjusted p-value (FDR $<0.1)$. Positive $\beta_{\text {state }}$ values represent elevated in OVOB-Fasted and negative $\beta_{\text {state }}$ values represent elevated in OVOB-Random-Fed.

In OVOB individuals, metabolites were identified that are associated with IR, measured by HOMA-IR (Figure S2e). Sex stratified models were run considering differences in glucose homeostasis measures by sex. Linear regression models were run separately at the Fasted Visit ( $\mathrm{t} 0$, $\mathrm{t} 60$, fold change) and the Random-Fed Visit ( $\mathrm{t} 0$, $\mathrm{t} 60$, fold change) on metabolite levels ( $\log 2$ and standardized across each time and state), adjusting for age at the Fasted Visit.

$$
\text { HOMA }-\mathrm{IR}=\beta_{\mathrm{o}}+\beta_{\text {metabolite }} X+\beta_{\text {age }} \mathrm{X}+\epsilon_{\mathrm{i}}
$$

Differential metabolites were identified using an adjusted $p$-value (FDR $<0.1)$. All statistical analyses were performed in $\mathrm{R}$ version 4.0.

\section{Results}

\subsection{Participant Characteristics}

Descriptive characteristics of the study population from the Fasted OGTT Challenge are reported in Table 1. Most of the cohort was Caucasian and non-Hispanic and included more females $(n=160)$ than males $(n=123)$, with similar distributions of sex, race, and ethnicity between OVOB and lean groups. No group trend was observed between IGT, IFG, and prediabetes status, with $15 \%$ of OVOB and $12 \%$ of lean having prediabetes. Three of the six lean participants who classified as prediabetic had a BMI percentile of $84 \%$, potentially explaining why group trends were not observed. No group differences were observed in glucose levels during the OGTT (Figure 2) or glucose response measured by AUC glucose. Group differences in the insulin response to the OGTT were observed, with a larger insulin response, measured by AUC insulin, and higher insulin levels beginning at t0 and continuing through the completion of blood draws in OVOB (Figure 2). Sex differences were observed with higher levels of glucose t120 $(p=0.024)$, insulin $\mathrm{t} 90(p=0.002)$, insulin t120 $(p<0.001)$, and BMI percentile $(p=0.007)$ in females and higher levels of glucose t30 $(p=0.003)$ in males (Table S1). Females had a larger insulin response to the OGTT than males, measured by insulin AUC ( $p=0.016)$ (Figure S3), suggesting a small decrease in insulin sensitivity. 
Table 1. Characteristics of study participants at the Fasted Visit, stratified by weight group. Significance denoted with unadjusted $p$-value $<0.05$ (bolded).

\begin{tabular}{|c|c|c|c|}
\hline Categorical Variables & $\begin{array}{c}\text { OVOB } \\
n(\%)\end{array}$ & $\begin{array}{l}\text { Lean } \\
n(\%)\end{array}$ & $p$-Value ${ }^{1}$ \\
\hline \multicolumn{4}{|l|}{ sex } \\
\hline Male & $97(43 \%)$ & $26(47 \%)$ & \multirow[b]{2}{*}{0.5254} \\
\hline Female & $131(57 \%)$ & $29(53 \%)$ & \\
\hline \multicolumn{4}{|l|}{ race } \\
\hline Asian/Pacific Islander & $4(2 \%)$ & $4(7 \%)$ & \multirow{5}{*}{0.1745} \\
\hline African American/Black & $59(26 \%)$ & $12(22 \%)$ & \\
\hline White & $135(59 \%)$ & $32(58 \%)$ & \\
\hline more than one race & $19(8 \%)$ & $6(11 \%)$ & \\
\hline did not wish to report & $11(5 \%)$ & $1(2 \%)$ & \\
\hline \multicolumn{4}{|l|}{ ethnicity } \\
\hline Hispanic & $18(8 \%)$ & $5(9 \%)$ & \multirow[b]{2}{*}{0.7707} \\
\hline non-Hispanic & $210(92 \%)$ & $50(91 \%)$ & \\
\hline \multicolumn{4}{|c|}{ abnormal 2-hr plasma glucose $(\geq 140 \mathrm{mg} / \mathrm{dL})$} \\
\hline Yes & $16(7 \%)$ & $3(5 \%)$ & \multirow[b]{2}{*}{0.6776} \\
\hline No & $212(93 \%)$ & $52(95 \%)$ & \\
\hline \multicolumn{4}{|c|}{ abnormal fasting plasma glucose $(\geq 100 \mathrm{mg} / \mathrm{dL})$} \\
\hline Yes & $8(4 \%)$ & $1(2 \%)$ & \multirow[b]{2}{*}{0.5213} \\
\hline No & $220(96 \%)$ & $54(98 \%)$ & \\
\hline \multicolumn{4}{|c|}{ ADA prediabetes (FPG $\geq 100 \mathrm{mg} / \mathrm{dL}$ or $2-\mathrm{hr}$ PG $\geq 140 \mathrm{mg} / \mathrm{dL}$ or $\mathrm{HbA} 1 \mathrm{c} \geq 5.7 \%$ ) } \\
\hline Yes & $35(1 \overline{5} \%)$ & $6(12 \%)$ & \multirow[b]{2}{*}{0.4009} \\
\hline No & $193(85 \%)$ & $49(88 \%)$ & \\
\hline Continuous Variables & $\begin{array}{c}\text { OVOB } \\
\text { Mean (SD) }\end{array}$ & $\begin{array}{c}\text { Lean } \\
\text { Mean (SD) }\end{array}$ & $p$-Value ${ }^{2}$ \\
\hline age (years) & $12.9(2.5)$ & $13.0(2.6)$ & 0.7301 \\
\hline BMI percentile & $95(4)$ & $59(27)$ & $7.52^{-14}$ \\
\hline HOMA-IR & $5.13(2.99)$ & $2.80(1.21)^{3}$ & $4.53^{-8}$ \\
\hline $\mathrm{HbA} 1 \mathrm{c}$ & $5.2(0.3)$ & $5.1(0.3)^{3}$ & 0.2082 \\
\hline fast time (hours) & $14.0(1.3)$ & $14.1(1.4)$ & 0.7606 \\
\hline \multicolumn{4}{|l|}{ fasting OGTT response } \\
\hline glucose (t0) (mg/dL) & $84(8)$ & $85(8)$ & 0.7475 \\
\hline glucose $(\mathrm{t} 30)(\mathrm{mg} / \mathrm{dL})$ & $126(22)$ & $132(25)$ & 0.1119 \\
\hline glucose $(\mathrm{t} 60)(\mathrm{mg} / \mathrm{dL})$ & $112(29)$ & $117(26)$ & 0.2182 \\
\hline glucose $(\mathrm{t} 90)(\mathrm{mg} / \mathrm{dL})$ & $107(26)$ & $106(21)$ & 0.6263 \\
\hline glucose $(\mathrm{t} 120)(\mathrm{mg} / \mathrm{dL})$ & $102(24)$ & $98(22)$ & 0.2562 \\
\hline insulin $(\mathrm{t} 0)(\mu \mathrm{U} / \mathrm{mL})$ & $24(14)$ & $13(5)^{3}$ & $1.55 \times 10^{-8}$ \\
\hline insulin $(\mathrm{t} 30)(\mu \mathrm{U} / \mathrm{mL})$ & $194(132)$ & $112(72)$ & $4.09 \times 10^{-9}$ \\
\hline insulin $(\mathrm{t} 60)(\mu \mathrm{U} / \mathrm{mL})$ & $156(117)$ & $87(53)$ & $7.04 \times 10^{-10}$ \\
\hline insulin $(\mathrm{t} 90)(\mu \mathrm{U} / \mathrm{mL})$ & $145(126)$ & $80(56)$ & $2.69 \times 10^{-8}$ \\
\hline insulin $(\mathrm{t} 120)(\mu \mathrm{U} / \mathrm{mL})$ & $133(117)$ & $65(57)$ & $2.42 \times 10^{-9}$ \\
\hline AUC glucose & $3121(2112)$ & $3294(1711)$ & 0.5228 \\
\hline AUC insulin & $17,223(11,206)$ & $9648(5468)$ & $1.33 \times 10^{-11}$ \\
\hline
\end{tabular}

\footnotetext{
${ }^{1}$ Represents Pearson's chi-square test for categorical variables. ${ }^{2}$ Represents unpaired $t$-test for continuous variables. ${ }^{3} n=54$.
} 

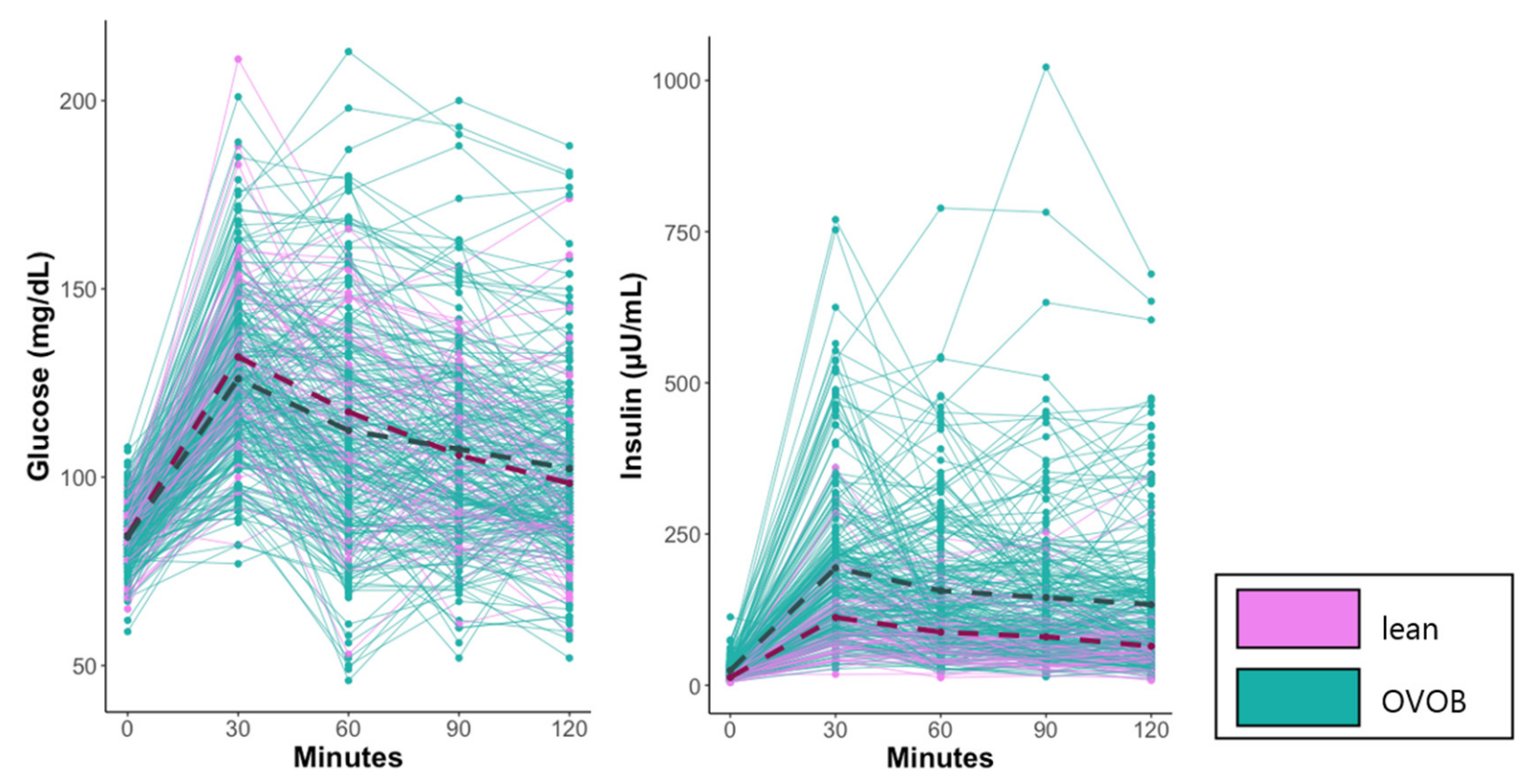

Figure 2. Kinetics of blood glucose and insulin response to the glucose test at the Fasted Visit. OVOB and lean participants arrived fasted prior to the consumption of the glucose challenge. Blood glucose and insulin were profiled before and during the OGTT. Mean values reported for lean (dark pink dash) and OVOB (dark slate gray dash). OVOB, overweight and obese.

\subsection{Influence of Obesity and Sex on the Fasting Metabolome}

At the Fasted Visit, 66 metabolites were significantly associated with BMI group (OVOB vs. lean) at t0, adjusting for sex and age (Table S2). Select differential metabolites by BMI group are shown in Figure 3. Short-chain ACs were elevated in OVOB compared to lean, including AC 3:0, 5:0, and 5:0-OH, representing alterations in BCAA metabolism. No differences were observed in BCAAs, potentially because our analysis did not account for muscle mass differences between OVOB and lean [7]. The aromatic amino acid tryptophan and its metabolite kynurenine was significantly elevated in OVOB, in contrast to the literature that consistently shows phenylalanine and tyrosine being elevated with obesity [27]. The biomarker 3-indolepropionate, a tryptophan metabolite that has been associated with a reduced likelihood of developing T2D [28], was significantly elevated in lean participants. Very long-chain FAs were elevated in lean at t0, with no BMI group differences observed in long-chain FAs. In lean participants, there were higher levels of beta-oxidation AC intermediates (AC 10:0, AC12:0, AC 14:1, AC 14:2, AC 16:0, and AC 18:2) and omega oxidation dicarboxylic FAs (FA 10:0-COOH, FA 11:0-COOH, and FA 16:0-COOH), potentially suggesting increased flux through FA oxidation pathways in lean individuals at fasting. Several lysophospholipids were elevated in lean compared to OVOB at t0, including lysophosphocholine (LPC) 16:0, LPC 17:0, LPC 18:1, LPC 18:2, LPC 20:0, and LPE 18:2, which parallels studies in pediatrics [8] and adults [29]. Multiple lipid species, including diglycerides (DG), phosphocholine (PC), and sphingomyelin (SM), were elevated in OVOB individuals, due to elevation in fat mass and consistent with previous observations [7]. Chenodeoxycholate (CDCA), a primary bile acid synthesized in the liver, was elevated in OVOB at t0. Multiple conjugated bile acids were differential between BMI groups at $\mathrm{t} 0$, including glycocholate, taurocholate, and tauro-alpha/beta-muricholate were elevated in lean and hyodeoxycholate was elevated in OVOB. 

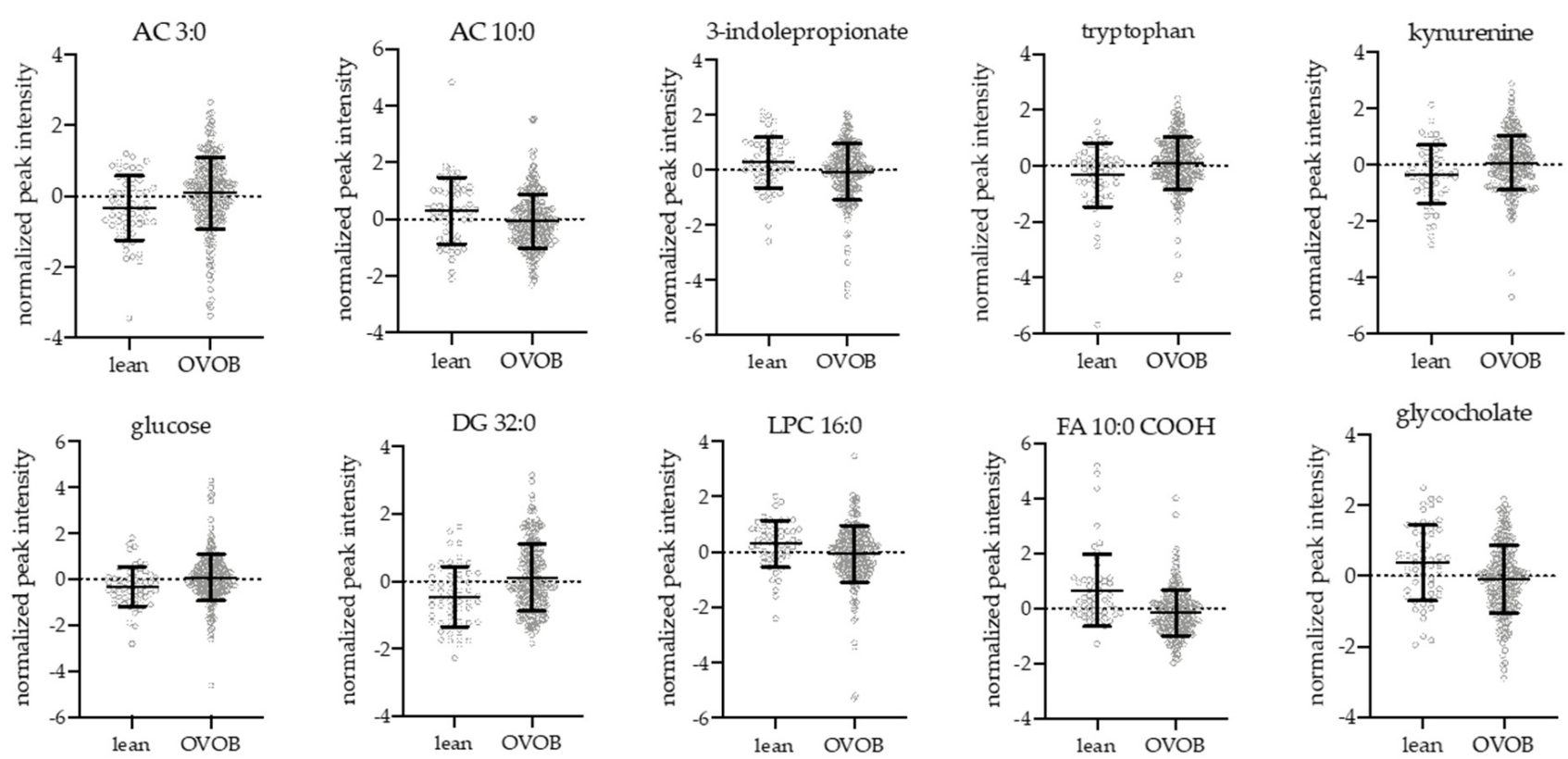

Figure 3. Differential metabolites between OVOB and lean participants during the Fasted OGTT Challenge. Linear regression analyses identified metabolites associated with the OVOB and lean groups at $\mathrm{t} 0$ ( $\beta_{\text {group }}$ ), adjusting for sex and age (FDR < 0.1, 66 metabolites). Selected differential metabolites represent metabolic pathways associated with obesity at $\mathrm{t} 0$. Normalized peak intensities and standard deviations are reported.

Metabolites significantly associated with BMI group at t0 (FDR $<0.1)$ were selected for metabolite set enrichment analysis (MSEA) to identify biological pathways enriched with obesity (Figure S4). No pathways reached an adjusted significance threshold, although Beta Oxidation of Very Long Chain Fatty Acids was trending towards significantly enriched at t0 (unadjusted $p=0.07$ ).

As sex is associated with BMI percentile and glucose homeostasis measures within this cohort (Table S1), metabolites were identified that were differential by sex at $\mathrm{t} 0$ during the Fasted Visit. Using unpaired $t$-tests, 40 metabolites were associated with sex $($ FDR $<0.05)$, with higher levels of FA, SM, and PCs in females and higher levels of short-chain ACs and amino acid metabolites (e.g., kynurenine and 3-methyl-2-oxovalerate) in males (Table S3). These associations emphasize variations in fat and muscle mass in adolescents during puberty, as previous findings detail differential metabolites elevated in obese males and females [7]. Sex-stratification will be considered in additional analyses.

\subsection{Metabolome Response to the OGTT in OVOB and Lean Participants}

The response of the metabolome to an OGTT is represented in Figure 4, stratifying individuals by state (Fasted and Random-Fed), time ( $\mathrm{t} 0$ and t60), and group (lean and OVOB). Metabolites peak intensities were centered across all samples and metabolites were grouped using hierarchical clustering to identify groups of metabolites with similar dynamics during the OGTT. Significant changes in metabolite levels from to to t60 are reported, including alterations in $68 \%$ of metabolites in lean during the Fasted OGTT Challenge (Figure S5a), $84 \%$ of metabolites in OVOB during the Fasted OGTT Challenge (Figure S5b), and 77\% of metabolites in OVOB during the Random-Fed OGTT Challenge (Figure S5c) (FDR < 0.05). Most metabolites decreased in response to the OGTT, which may be attributed to the high abundance of lipids within the annotated metabolites in this dataset. Metabolite classes that consistently decreased in response to the OGTT include medium- and long-chain ACs, FFA, and lipids, such as SMs, PCs, and DGs. The metabolite with the largest increase was hippurate, increasing at approximately $4 \log 2 \mathrm{FC}$ in each group. As reported by Shaham et al. [2], this likely reflects the metabolism of the preservative 
benzoic acid, found in the glucola beverage used for the OGTT [30]. All paired $t$-tests are reported in Table S4.

Using a fold change, differences in metabolite response between BMI groups at the Fasted Visit was explored, identifying 15 significant metabolites (Figure S6). Five medium- and long-chain ACs (AC 8:0, 10:1, 12:1, 14:2, and 16:1), two fatty acid oxidation intermediates (FA 10:0-COOH and FA 16:0-COOH), and six FAs (FA 12:0, 12:2, 14:2, 20:0, 20:1, and 22:1) had a larger decrease in lean than OVOB. These results suggest a more robust decrease in lipolysis and beta-oxidation in lean in response to the glucose challenge.
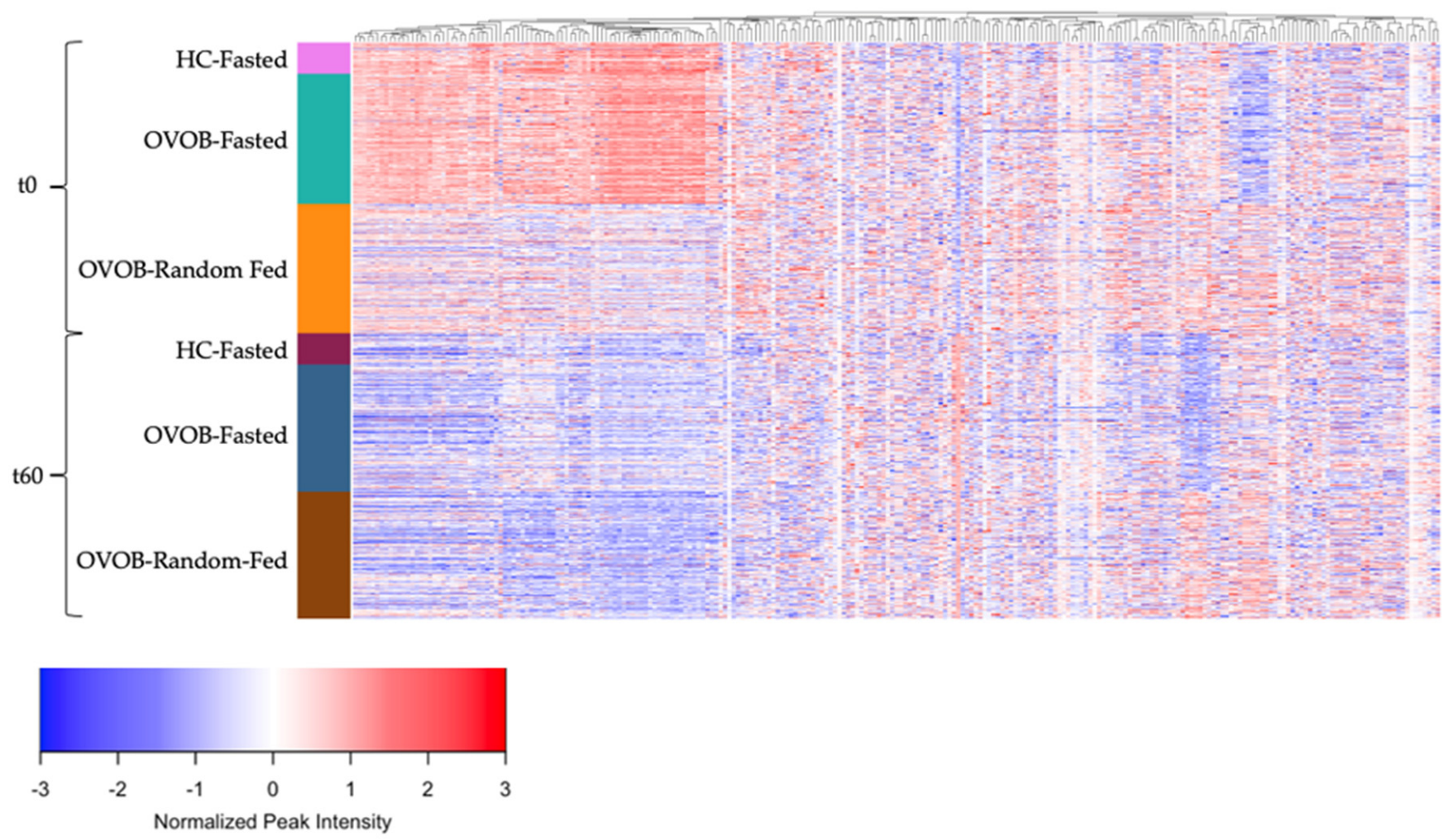

Figure 4. Dynamic response of the metabolome to an oral glucose tolerance test. Heatmap of normalized peak intensity for individual metabolites $(p=246$ ) (mean 0 , standard deviation 1). Metabolites are grouped by hierarchical clustering (columns). Subjects are ordered by group (OVOB and lean), time point after the OGTT (t0 and t60), and state (Fasted and Random-Fed) (rows). OVOB, overweight and obese.

\subsection{Metabolome Differences between the Fasted and Random-Fed OGTT Challenge in OVOB}

OVOB participants returned to the clinic for a random-fed state OGTT challenge. At t0, random-fed OVOB participants had significantly higher glucose $(p=0.0052)$ and insulin levels $\left(p=3.28^{-20}\right)$ than individuals in the fasted state (Table S5). At t60, random-fed participants had significantly lower levels of glucose $\left(p=1.40^{-21}\right)$ than individuals in the Fasted OGTT Challenge, although their insulin levels were not significantly different $(p=0.657)$. These results suggest individuals arriving to the clinic for the OGTT in a variety of fed states have a primed insulin response, enabling the rapid response to the glucose load. Metabolites were identified that were associated with arriving to the OGTT Challenge fasted or random-fed at $\mathrm{t} 0$ and $\mathrm{t} 60$. At $\mathrm{t} 0,155$ metabolites (63\% profiled) and at t60, 122 metabolites (49\% profiled) differed between OVOB-Fasted and OVOB-Random-Fed (Table S6). Grouping by super pathway, metabolites are represented indicating direction of association ( $\left.\beta_{\text {state }}\right)$ and significance $(-\log 10[p$-value $])$ for t0 (Figure $\left.5 \mathrm{~A}\right)$ and t60 (Figure $\left.5 \mathrm{~B}\right)$. 


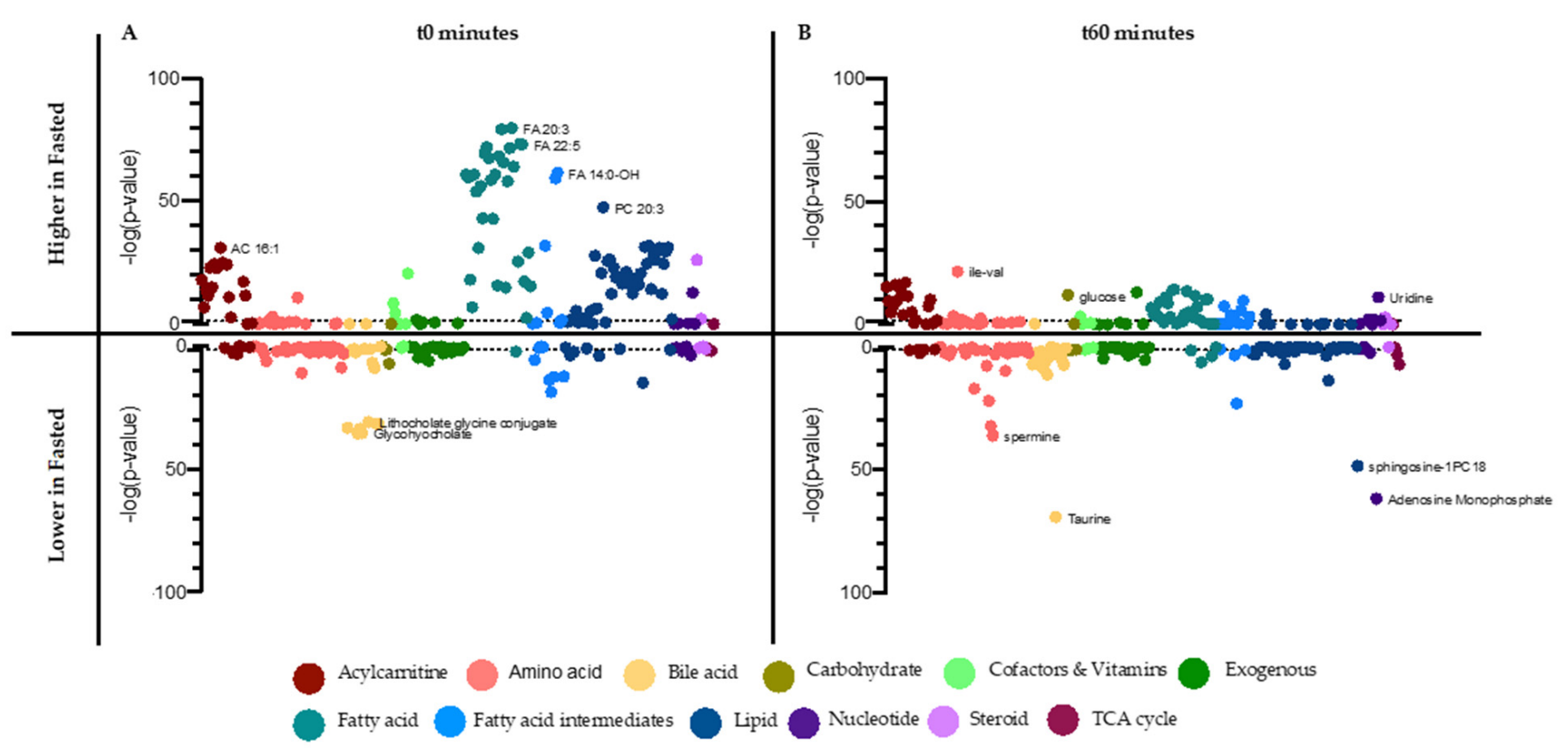

Figure 5. Differential metabolites between Fasted and Random-Fed in OVOB. Linear regression analyses identified metabolites associated with OVOB-Fasted and OVOB-Random-Fed (Bstate) at (A) t0 and (B) t60. On the top of the plots, metabolites are reported that are higher in OVOB-Fasted (Bstate $<0)$, with position indicated by $-\log 10(p$-value). On the bottom of the plots, metabolites are reported that are lower in OVOB-Fasted (Bstate $>0$ ), with position indicated by $-\log 10$ ( $p$-value). Metabolites are listed in the same order in 5A and 5B. Colors indicate metabolite class. Horizontal lines (dotted) signify FDR $=0.1$. Several distinguishing metabolite names are listed. Age and sex are included in the model.

At t0, almost all the FAs profiled (97\%) were significantly higher at the Fasted Visit compared to the Random-Fed Visit, as expected, representing mobilization of energy substances (anabolism) from adipose tissue during fasting. In parallel, additional lipids were higher at the Fasted Visit, including all SMs and 94\% of the profiled PCs (15 PCs). Lysophospholipids varied in their associations with state, with five higher in the fasted state (LPC 16:0, LPC 17:0, LPC 18:1, LPC 20:0, LPC 23:0) and two higher in the random state (LPC 18:2 and LPE 18:2). Most medium- and long-chain ACs were higher at fasting, paralleling the FA levels and representing increases in beta-oxidation at fasting [31]. Interestingly, three of the four dicarboxylic fatty acids profiled were higher at the Random-Fed state at (FA 9:0$\mathrm{COOH}$, FA 10:0-COOH, and FA 11:0- $\mathrm{COOH}$ ), suggesting increased omega oxidation related to the fed state, perhaps due to increased carbohydrate oxidation reducing the capacity to oxidize FA still entering the system. Many amino acids were higher at the Random-Fed Visit, including histidine, isoleucine/leucine, methionine, proline, tryptophan, tyrosine, and valine. Several short-chain ACs were higher in at the Random-Fed Visit, indicating increased BCAA metabolism. Bile acids, including primary, secondary, and conjugate bile acids, were higher at the Random-Fed Visit, representing bile acid and gut hormone response to a meal [32].

At t60, the metabolome represents the switch from an anabolic to a catabolic state in response to the glucose challenge. A portion of the differential metabolites at t0 normalized between OVOB-Fasted and OVOB-Random-Fed, including most of the differential lipids (SMs and PCs). At t60, 75\% of the FAs and 78\% of the medium- and long-chain ACs remain higher at the Fasted Visit. Fatty acid oxidation intermediates varied in their association with state at t60, with several higher in the Fasted group (FA 9:0-COOH, FA 12:0-OH, FA 12:0-NH2, and FA 14:0-OH) and several higher in the Random-Fed group (FA 10:0-OH and FA 10:0-COOH). Seven of the twelve lysophospholipids profiled were higher at the Random-Fed Visit at t60. Amino acids and bile acids remained higher in Random-Fed at $\mathrm{t} 60$. 


\subsection{Sex-Specific Associations of Metabolite Trajectories with Insulin Resistance in Participants with Overweight and Obesity}

In $\mathrm{OVOB}$, metabolites were identified from the fasted ( $\mathrm{t} 0$, $\mathrm{t} 60$, and fold change) and random-fed ( $\mathrm{t} 0, \mathrm{t} 60$, and fold change) glucose challenges that were associated with IR, measured by HOMA-IR (Figure S7). Sex stratified linear models were used considering the differential glucose and insulin responses between males and females (Table S1). All results are reported in Table S7.

In males across all visits and time points, no metabolites were significantly associated with HOMA-IR. The metabolite mesobilirubinogen was trending towards positive association with HOMA-IR at 160 in the Fasted and Random-Fed visit (FDR < 0.2).

In females, metabolites within multiple pathways were correlated with HOMA-IR at t0 and t60 during the Fasted and Random-Fed visits (Table 2). Consistently, diacylglycerides (DG 32:0, DG 32:1, DG 34:1, and DG 34:2) and the nucleotide urate were positively associated with HOMA-IR, the latter supported by previous work establishing the connection between hyperuricemia and IR [33]. More specifically, at t60 during the fasted visit, several amino acid metabolites (isoleucine/leucine, AC 5:0-OH, proline, and glutamate) and lipids (DGs and PCs) were positively associated with HOMA-IR. These results expand upon previous work [3], which found a blunted decrease in levels of BCAAs and other amino acid metabolites in subjects with IR. Represented by the fold change from the fasted visit, medium- and long-chain ACs were positively associated with HOMA-IR, demonstrating that a blunted decline in ACs in response to a glucose challenge is associated with IR, which parallels the decline in FA following the glucose challenge. Comparing the significant metabolites at Fasted t60 vs. the fold change during the fasted visit, only DG 32:0 and PC 32:1 were significantly associated with HOMA-IR in both models. Although less metabolites were significantly associated with HOMA-IR during the Random-Fed Visit, at t60, DGs, monoglycerides, glutamate, and urate exhibited positive associations. No significant associations were observed using the fold change from the Random-Fed.

Table 2. Metabolites associated with HOMA-IR in females with overweight and obesity at the Fasted and Random-Fed Visit. Beta coefficients and standard errors from linear regression models are reported, adjusting for age at the Fasted Visit $(\mathrm{FDR}<0.1)$.

\begin{tabular}{|c|c|c|c|c|c|c|}
\hline Metabolite & Pathway & Fasted to & Fasted t60 & $\begin{array}{l}\text { Fasted Fold } \\
\text { Change }\end{array}$ & $\begin{array}{l}\text { Random-Fed } \\
\text { t0 }\end{array}$ & $\begin{array}{c}\text { Random-Fed } \\
\text { t60 }\end{array}$ \\
\hline AC 12:0 & acylcarnitine & & & $2.0 \pm 0.3$ & & \\
\hline AC 12:1 & acylcarnitine & & & $2.7 \pm 0.5$ & & \\
\hline AC 14:0 & acylcarnitine & & & $2.3 \pm 0.8$ & & \\
\hline AC 16:0 & acylcarnitine & & & $2.6 \pm 0.6$ & & \\
\hline AC $16: 1$ & acylcarnitine & & & $1.3 \pm 0.7$ & & \\
\hline AC 18:0 & acylcarnitine & & & $1.6 \pm 0.2$ & & \\
\hline AC 5:0-OH & acylcarnitine & & $0.9 \pm 0.3$ & & & \\
\hline AC $5: 1$ & acylcarnitine & & & $1.5 \pm 1.6$ & & \\
\hline AC 6:0 & acylcarnitine & & & $1.7 \pm 0.6$ & & \\
\hline gamma-glutamyltyrosine & amino acid & $0.8 \pm 0.2$ & $0.9 \pm 0.3$ & & $0.9 \pm 0.3$ & \\
\hline Glu-Phe & amino acid & & $0.9 \pm 0.2$ & & & \\
\hline glutamate & amino acid & & $0.7 \pm 0.3$ & & & $0.9 \pm 0.3$ \\
\hline indole-3-methyl acetate & amino acid & & $0.7 \pm 0.3$ & & & \\
\hline $\begin{array}{l}\text { L-gamma- } \\
\text { glutamylisoleucine }\end{array}$ & amino acid & & $0.8 \pm 0.3$ & & & \\
\hline Leu-Ile & amino acid & & & $0.7 \pm 0.5$ & & $0.9 \pm 0.2$ \\
\hline leucine+isoleucine & amino acid & & $0.7 \pm 0.3$ & & & \\
\hline $\mathrm{N}$-acetylphenylalanine & amino acid & & $0.7 \pm 0.3$ & & & \\
\hline Phe-Phe & amino acid & & & $-0.7 \pm 0.3$ & & \\
\hline
\end{tabular}


Table 2. Cont.

\begin{tabular}{|c|c|c|c|c|c|c|}
\hline Metabolite & Pathway & Fasted t0 & Fasted t60 & $\begin{array}{l}\text { Fasted Fold } \\
\text { Change }\end{array}$ & $\begin{array}{c}\text { Random-Fed } \\
\text { t0 }\end{array}$ & $\begin{array}{c}\text { Random-Fed } \\
\text { t60 }\end{array}$ \\
\hline Phe-Trp & amino acid & & $0.7 \pm 0.3$ & & & \\
\hline pipecolate & amino acid & & & $-0.6 \pm 1.1$ & & \\
\hline proline & amino acid & & $0.7 \pm 0.3$ & & & \\
\hline cholate & bile acid & & $0.8 \pm 0.3$ & & & \\
\hline hyocholate & bile acid & & $0.8 \pm 0.3$ & & & \\
\hline indole-3-lactate & carbohydrate & & $0.7 \pm 0.3$ & & & \\
\hline caffeine & exogenous & & & $1.7 \pm 0.6$ & & \\
\hline FA $18: 4$ & fatty acid & & $0.8 \pm 0.3$ & & & \\
\hline FA $20: 3$ & fatty acid & & & $1.1 \pm 0.3$ & & \\
\hline FA 22:1 & fatty acid & & & $1.9 \pm 0.5$ & & \\
\hline 3-hydroxyphenyl-valerate & $\begin{array}{c}\text { fatty acid } \\
\text { intermediate }\end{array}$ & & & $0.9 \pm 0.6$ & & \\
\hline DG 32:0 & lipid & $1.2 \pm 0.2$ & $1.2 \pm 0.2$ & $2.2 \pm 0.4$ & $1.1 \pm 0.2$ & $1.0 \pm 0.2$ \\
\hline DG 32:1 & lipid & $1.0 \pm 0.2$ & $1.1 \pm 0.2$ & & $0.9 \pm 0.2$ & $0.8 \pm 0.2$ \\
\hline DG 34:1 & lipid & & $1.0 \pm 0.2$ & & $0.9 \pm 0.2$ & $0.9 \pm 0.2$ \\
\hline DG 34:2 & lipid & $1.1 \pm 0.2$ & $1.0 \pm 0.2$ & & $0.9 \pm 0.2$ & $0.8 \pm 0.2$ \\
\hline DG 36:2 & lipid & & $0.7 \pm 0.2$ & & & \\
\hline DG 36:3 & lipid & & $0.7 \pm 0.3$ & & & \\
\hline MG 14:0 & lipid & & & & & $1.0 \pm 0.2$ \\
\hline MG 16:0 & lipid & & & $1.2 \pm 0.3$ & & \\
\hline MG 18:1 & lipid & $0.8 \pm 0.2$ & $0.9 \pm 0.2$ & & & $0.7 \pm 0.3$ \\
\hline LPC 16:0 & lipid & & & $1.1 \pm 1.2$ & & \\
\hline LPC 18:2 & lipid & & & $0.8 \pm 1.7$ & & \\
\hline PC 32:1 & lipid & & $0.8 \pm 0.2$ & $1.0 \pm 0.4$ & & \\
\hline PC $34: 3$ & lipid & & $0.7 \pm 0.3$ & & & \\
\hline PC 34:4 & lipid & & $0.7 \pm 0.3$ & & & \\
\hline $\begin{array}{l}\text { N2,N2- } \\
\text { dimethylguanosine }\end{array}$ & nucleotide & & $0.7 \pm 0.3$ & & & \\
\hline urate & nucleotide & $0.8 \pm 0.2$ & $1.0 \pm 0.2$ & & $1.0 \pm 0.2$ & $0.9 \pm 0.2$ \\
\hline
\end{tabular}

\section{Discussion}

In the present study, we have characterized the metabolome response during an OGTT in OVOB $(n=228)$ and lean adolescents $(n=55)$. We identified metabolites that change significantly during the glucose challenge, highlighting the switch from FA to glucose oxidation at $60 \mathrm{~min}$ during the OGTT. We classified differential metabolites by BMI status at baseline and during the OGTT, suggesting that at an early age, obesity and its metabolic consequences may influence the metabolome dynamics in response to a challenge. Subsequently, overweight and obese adolescents returned to the clinic for a random-fed glucose challenge to compare the fasted and random-fed metabolome to degree of IR, and significant associations were found in female participants but not in males. Our results are the first study to deeply assess the fasted and random-fed metabolome response in adolescents and will be used for future analyses predicting the longitudinal risk of prediabetes development within the cohort.

\subsection{Lipids, Fatty Acids, and Acylcarnitines}

In response to the glucose challenge, most lipids, FAs, and FA oxidation intermediates, including hydroxyl-FAs, dicarboxylic FAs, and acylcarnitines, decreased. As observed in previous studies in adults, these alterations in the metabolome are reflective of the switch from FA oxidation to glucose oxidation and fat storage during the OGTT [34]. Acylcarnitines, biomarkers of mitochondrial beta-oxidation, reflecting the relative utilization of FA to carbohydrate [34] and reflect the degree of IR [35]. At the Fasted-Visit, several mediumand long-chain ACs and dicarboxylic FAs were lower in OVOB participants (Figure 3) and had a blunted decline in OVOB participants (Figure S6). Furthermore, in OVOB females at 
the fasted visit, the fold change of eight ACs (AC 5:1, 6:0, 12:0, 12:1, 14:0, 16:0, 16:1, and 18:0) was positively associated with HOMA-IR (FDR $<0.1)$. These results suggest that starting at a young age, obesity and IR influences metabolic flexibility in response to a glucose load [36]. In parallel, Nowak et al. [19] in a group of older males observed that AC 10:0 and AC 12:0 exhibited a smaller decline at $30 \mathrm{~min}$ in response to an OGTT, suggesting that the sustained elevation of the AC may directly impair insulin sensitivity. Our findings suggest that during adolescence, the prolonged insulin response (Figure 2) in OVOB females is also associated with insulin resistance.

At the fasted visit at baseline, lipids, including DGs and SMs, exhibited positive associations with obesity (Table S3), supported by previous analyses [7]. A primary question in these studies is whether the non-fasted state could be used to identify changes in metabolism in relation to IR. In females, at both the Fasted and Random-Fed Visit at t0 and t60, diacylglycerides (DG 32:0, DG 32:1, DG 34:1, and DG 34:2) were positively associated with HOMA-IR, suggesting that independent of fed-state, these lipids may provide predictive ability for the progression of IR and T2D.

\subsection{Amino Acids}

In lean adolescents, approximately half of the profiled amino acids and their metabolites decreased in response to the OGTT, including leucine/isoleucine, methionine, histidine, serine, and glutamate, representing a decrease in proteolysis [2,3]. Deviations in amino acids response were observed between OVOB and lean, potentially due to the elevated insulin response within OVOB (Table 1, Figure 2). The larger insulin response in OVOB may act on skeletal muscles to decrease protein degradation [37], as evidenced by significant decreases in amino acids, including trypthophan, lysine, and glutamine, in only OVOB adolescents at t60. Comparing the metabolome response to an OGTT in 14 obese and 6 lean adults, Geidenstam et al. [10] observed at 30 min post-OGTT that asparagine, glutamate, taurine, tyrosine, and leucine/isoleucine increased in obese adults, which was absent in lean. This effect was not evident in our cohort, potentially because the metabolome was profiled at a later timepoint (t60).

At t60 during the fasted visit, several amino acids (leucine/isoleucine, glutamate, and proline) and amino acid metabolites (gamma-glutamyltyrosine, L-gamma-glutamylisoleucine and N-acetylphenylalanine) were associated with IR in females (Table 2). Without stratifying by sex, Mullner et al. observed levels of BCAAs associated with a heightened insulin response [11]. Frequent inconsistencies in the association between BCAA and IR in adolescents are observed [7,38], due to study population differences in age, sexual maturation, and degree of IR, representing major challenges in paediatric prediction studies. Glutamate was associated with HOMA-IR in the Fasted and Random-Fed OGTT at t0 and t60. In a rate-limiting TCA cycle step, alpha-ketoglutarate is converted to glutamate-by-glutamate dehydrogenase, allowing for a rescue pathway of excess TCA substrate. Elevated levels of glutamate have been associated with an increased risk of T2D [39], as our results highlight the sensitivity between IR and TCA cycle overload in females. Overall, the t60 metabolome at the Fasted Visit had the largest number of significant amino acids with IR in females, suggesting a lack of suppression of proteolysis with reduced insulin sensitivity.

\subsection{Bile Acids}

Paralleling previous studies [2,34], we observed a dramatic increase in several bile acids in response to the glucose challenge, including glycocholate, glycodeoxycholate, glycohyocholate, and taurocholate. In response to a meal, the gallbladder releases bile into the small intestine, stimulated by gastric filling and the intestinal hormone cholecystokinin (CCK). Post intestinal absorption and transport to the liver, it is estimated that $10-30 \%$ of bile acids reach systemic circulation [40]. Our results and others [2,34] have suggested that a bolus of glucose stimulates the release of bile acids in the gallbladder, supported by Liddle et al. finding that glucose ingesting stimulates CCK production [41]. Previous work has suggested a link between bile acid secretion and metabolism with obesity and 
IR [42]. At baseline, during the Fasted Visit, several primary and secondary bile acids were associated with obesity, including positive associations with chenodeoxycholate, hyodeoxycholate, and deoxycholate and inverse associations with glycocholate, glycohyocholate, taurocholate, and tauro-alpha/beta-muricholate. Furthermore, at t60 during the Fasted Visit, cholate and hyocholate were positively associated with IR in females (Table 2). Therefore, a blunted decrease of certain bile acids may be associated with insulin resistance and metabolic dysfunction.

\subsection{Conclustions and Future Directions}

The metabolome response to an OGTT may be associated with IR in a sex-specific manner, due to the observed differences in insulin response to an OGTT in adolescent males and females. In healthy and metabolically unhealthy youth, insulin sensitivity decreases during puberty [43]. Furthermore, in a cohort of healthy children and adolescents, girls in late puberty (Tanner's Stage 4 or 5) have higher insulin levels than boys [44]. The sexual dimorphism observed in late puberty is due, in part, to a higher growth hormone secretion in pubertal girls [45]. In our cohort, we observed higher levels of glucose t120, insulin t90, and insulin 120 in females, suggesting that females have a larger insulin response to the OGTT than males. Future analyses must be conducted to determine if the associations between the metabolome across visits and HOMA-IR in females is attributed to IR shifts during puberty or the onset of metabolic dysfunction and prediabetes.

The metabolome was comprehensively profiled using a liquid chromatography/mass spectrometry-based platform, generating approximately 250 metabolites. Our study utilized a well-powered sample size, strongly complementing and elaborating on the only other study assessing the metabolome response to an OGTT in adolescents [11] by incorporating both a fasted and a random-fed visit in the OVOB participants. Our results emphasize the potential of analyzing the metabolome response in a random glucose challenge for the prediction of metabolic dysfunction, particularly in females. The results from this study emphasize that the switch from FA to glucose metabolism in response to a glucose challenge is associated with obesity and insulin resistance. Future work will collect plasma samples in response to a glucose challenge at more timepoints, such as $30 \mathrm{~min}$, to assess more subtle changes in the metabolites, similarly to Zhao et al. [34].

Our study design presented two limitations regarding the Random-Fed Visit. Firstly, we only recruited OVOB participants for the visit, not allowing for a comparison between lean and OVOB. Secondly, we desired to simulate a random glucose challenge that is typically performed in practice for women being screened for gestational diabetes using $50-\mathrm{g}$ of glucose. The differences in grams of glucose solution administered between the Fasted and Random-Fed Visit create challenges in the direct comparison of the metabolome response. Our priority was to replicate what is being practiced in the clinic. Future work should compare the metabolome response in different fed-states utilizing the same glucose load. A bioinformatic limitation in the study was the inability to map individual significant metabolites to biological pathways using MSEA, due to many metabolites with the pathways not being profiled in the untargeted metabolomics platform and the lack of HMDBs for metabolites that were significant. Future directions will incorporate a partial correlation-based approach [46] to assess alterations in the relationship of metabolites at the fasted random-fed visit and if subnetworks of metabolites are associated with insulin resistance cross-sectionally and longitudinally.

Our results emphasize the utility of profiling multiple metabolic pathways outside glucose metabolism in understanding the associations between obesity, IR, and the response to a glucose challenge in adolescents. Classifying the metabolism of lipids, amino acids, and fatty acids, rather than solely glucose metabolism, deepens the understanding of the pathophysiology of insulin resistance in adolescents, with differences than adults due to pubertal development. Future work will test if the highlighted metabolic pathways complement or enhance the ability of glucose to predict the development of prediabetes during adolescence. 
Supplementary Materials: The following are available online at https: / www.mdpi.com/article/ 10.3390/nu13103365/s1, Figure S1: Oral glucose tolerance test specifications for the Fasted and Random-Fed Visits., Figure S2: Analysis objectives., Figure S3: Sex differences., Figure S4: Metabolic Set Enrichment Analysis between BMI groups at the Fasted OGTT Challenge., Figure S5: Trajectory of the metabolome between $\mathrm{t} 0$ and $\mathrm{t} 60 \mathrm{~min}$ within groups., Figure S6: Metabolite response differences between OVOB and lean participants during the Fasted OGTT Challenge., Figure S7: Distribution of HOMA-IR measures in OVOB participants, stratified by males and females., Table S1: Characteristics of study participants at the Fasted Visit, stratified by sex., Table S2: Metabolites associated with OVOB and lean participants at the Fasted Visit., Table S3: Metabolites associated with sex at the Fasted Visit., Table S4: Metabolite alterations in response to the OGTT within each group (lean and OVOB) and state (fasted and random-fed)., Table S5: Glucose and insulin levels at the Fasted and Random-Fed Visits for OVOB adolescents., Table S6: Metabolites differentially associated with the fasted and random-fed state in OVOB., Table S7: Sex stratified relationship between metabolites and HOMA-IR in OVOB.

Author Contributions: Conceptualization, J.L.L., E.H., C.F.B. and J.M.L.; methodology, E.H., T.S., M.K., J.W., W.D. and A.K.; formal analysis, J.L.L., J.Y.F., C.F.B. and J.M.L.; investigation, E.H. and J.M.L.; resources, C.F.B. and J.M.L.; data curation, E.H.; writing-original draft preparation, J.L.L. and C.F.B.; writing—review and editing, All authors; visualization, J.L.L. and J.Y.F.; supervision, C.F.B. and J.M.L.; project administration, E.H.; funding acquisition, C.F.B. and J.M.L. All authors have read and agreed to the published version of the manuscript.

Funding: This research was funded by the National Institute of Child Health \& Human Development (R01HD074559, JML) and this work utilized services supported by the Michigan Diabetes Research Center through the National Institute of Diabetes and Digestive and Kidney Diseases (P30DK020572), Michigan Institute for Clinical and Health Research through the National Institutes of Health (UL1TR000433 and UL1TR002240), and the Michigan Nutrition Obesity Research Center through the National Institutes of Health (DK089503). Dr. LaBarre was supported by the University of Michigan Training Program in Endocrinology and Metabolism (T32 DK007245). Dr. Lee is supported by the National Institute of Health (2P30DK089503-11 and UH3HD087979) and the Caswell Diabetes Institute at the University of Michigan. Dr. Burant is supported by The A. Alfred Taubman Medical Institute of the University of Michigan and the Robert C. and Veronica Atkins Foundation.

Institutional Review Board Statement: The study was conducted according to the guidelines of the Declaration of Helsinki and approved by the Institutional Review Board (or Ethics Committee) of The University of Michigan (HUM\#00006955 and initial date of approval 26 July 2007).

Informed Consent Statement: Informed consent or assent was obtained from all subjects involved in the study.

Data Availability Statement: Data described in the manuscript, code book, and analytic code will be made available upon request. Metabolomics data is available at the National Metabolomics Data Repository (metabolomicsworkbench.org/data/index. php).

Acknowledgments: We thank the staff of the Michigan Clinical Research Unit and Michigan Diabetes Research Center for their contributions to perform the study visits and laboratory measurements.

Conflicts of Interest: Medical advisory board for GoodRx (J.M.L.). No other conflicts to disclose.

\section{References}

1. Lascar, N.; Brown, J.; Pattison, H.; Barnett, A.H.; Bailey, C.J.; Bellary, S. Type 2 diabetes in adolescents and young adults. Lancet Diabetes Endocrinol. 2018, 6, 69-80. [CrossRef]

2. Shaham, O.; Wei, R.; Wang, T.J.; Ricciardi, C.; Lewis, G.D.; Vasan, R.S.; Carr, S.A.; Thadhani, R.; Gerszten, R.E.; Mootha, V.K. Metabolic profiling of the human response to a glucose challenge reveals distinct axes of insulin sensitivity. Mol. Syst. Biol. 2008, 4, 214. [CrossRef] [PubMed]

3. Ho, J.E.; Larson, M.G.; Vasan, R.S.; Ghorbani, A.; Cheng, S.; Rhee, E.P.; Florez, J.C.; Clish, C.B.; Gerszten, R.E.; Wang, T.J. Metabolite profiles during oral glucose challenge. Diabetes 2013, 62, 2689-2698. [CrossRef] [PubMed]

4. Diniz Behn, C.; Jin, E.S.; Bubar, K.; Malloy, C.; Parks, E.J.; Cree-Green, M. Advances in stable isotope tracer methodology part 1: Hepatic metabolism via isotopomer analysis and postprandial lipolysis modeling. J. Investig. Med. 2020, 68, 3-10. [CrossRef] 
5. $\quad$ Fukagawa, N.K.; Minaker, K.L.; Rowe, J.W.; Goodman, M.N.; Matthews, D.E.; Bier, D.M.; Young, V.R. Insulin-mediated reduction of whole body protein breakdown. Dose-response effects on leucine metabolism in postabsorptive men. J. Clin. Investig. 1985, 76, 2306-2311. [CrossRef] [PubMed]

6. Twig, G.; Zucker, I.; Afek, A.; Cukierman-Yaffe, T.; Bendor, C.D.; Derazne, E.; Lutski, M.; Shohat, T.; Mosenzon, O.; Tzur, D.; et al. Adolescent Obesity and Early-Onset Type 2 Diabetes. Diabetes Care 2020, 43, 1487-1495. [CrossRef]

7. LaBarre, J.L.; Peterson, K.E.; Kachman, M.T.; Perng, W.; Tang, L.; Hao, W.; Zhou, L.; Karnovsky, A.; Cantoral, A.; Tellez-Rojo, M.M.; et al. Mitochondrial Nutrient Utilization Underlying the Association Between Metabolites and Insulin Resistance in Adolescents. J. Clin. Endocrinol. Metab. 2020, 105. [CrossRef]

8. Butte, N.F.; Liu, Y.; Zakeri, I.F.; Mohney, R.P.; Mehta, N.; Voruganti, V.S.; Goring, H.; Cole, S.A.; Comuzzie, A.G. Global metabolomic profiling targeting childhood obesity in the Hispanic population. Am. J. Clin. Nutr. 2015, 102, 256-267. [CrossRef]

9. Perng, W.; Gillman, M.W.; Fleisch, A.F.; Michalek, R.D.; Watkins, S.M.; Isganaitis, E.; Patti, M.E.; Oken, E. Metabolomic profiles and childhood obesity. Obesity 2014, 22, 2570-2578. [CrossRef]

10. Geidenstam, N.; Spegel, P.; Mulder, H.; Filipsson, K.; Ridderstrale, M.; Danielsson, A.P. Metabolite profile deviations in an oral glucose tolerance test-a comparison between lean and obese individuals. Obesity 2014, 22, 2388-2395. [CrossRef] [PubMed]

11. Mullner, E.; Rohnisch, H.E.; von Bromssen, C.; Moazzami, A.A. Metabolomics analysis reveals altered metabolites in lean compared with obese adolescents and additional metabolic shifts associated with hyperinsulinaemia and insulin resistance in obese adolescents: A cross-sectional study. Metabolomics 2021, 17, 11. [CrossRef]

12. Lee, J.M.; Eason, A.; Nelson, C.; Kazzi, N.G.; Cowan, A.E.; Tarini, B.A. Screening practices for identifying type 2 diabetes in adolescents. J. Adolesc. Health 2014, 54, 139-143. [CrossRef]

13. Lee, J.M.; Gebremariam, A.; Wu, E.L.; LaRose, J.; Gurney, J.G. Evaluation of nonfasting tests to screen for childhood and adolescent dysglycemia. Diabetes Care 2011, 34, 2597-2602. [CrossRef]

14. International Diabetes Federation Guideline Development Group. Guideline for management of postmeal glucose in diabetes. Diabetes Res. Clin. Pr. 2014, 103, 256-268. [CrossRef] [PubMed]

15. Carayol, M.; Licaj, I.; Achaintre, D.; Sacerdote, C.; Vineis, P.; Key, T.J.; Onland Moret, N.C.; Scalbert, A.; Rinaldi, S.; Ferrari, P. Reliability of Serum Metabolites over a Two-Year Period: A Targeted Metabolomic Approach in Fasting and Non-Fasting Samples from EPIC. PLoS ONE 2015, 10, e0135437. [CrossRef] [PubMed]

16. Guasch-Ferre, M.; Hruby, A.; Toledo, E.; Clish, C.B.; Martinez-Gonzalez, M.A.; Salas-Salvado, J.; Hu, F.B. Metabolomics in Prediabetes and Diabetes: A Systematic Review and Meta-analysis. Diabetes Care 2016, 39, 833-846. [CrossRef] [PubMed]

17. Prasun, P. Role of mitochondria in pathogenesis of type 2 diabetes mellitus. J. Diabetes Metab. Disord. 2020, 19, 2017-2022. [CrossRef] [PubMed]

18. Grzych, G.; Chávez-Talavera, O.; Descat, A.; Thuillier, D.; Verrijken, A.; Kouach, M.; Legry, V.; Verkindt, H.; Raverdy, V.; Legendre, B.; et al. NASH-related increases in plasma bile acid levels depend on insulin resistance. J. Heptalogy Rep. 2020, $3,100222$. [CrossRef]

19. Nowak, C.; Hetty, S.; Salihovic, S.; Castillejo-Lopez, C.; Ganna, A.; Cook, N.L.; Broeckling, C.D.; Prenni, J.E.; Shen, X.; Giedraitis, V.; et al. Glucose challenge metabolomics implicates medium-chain acylcarnitines in insulin resistance. Sci. Rep. 2018, 8, 8691. [CrossRef]

20. Kuczmarski, R.J.; Ogden, C.L.; Grummer-Strawn, L.M.; Flegal, K.M.; Guo, S.S.; Wei, R.; Mei, Z.; Curtin, L.R.; Roche, A.F.; Johnson, C.L. CDC growth charts: United States. Adv. Data 2000, 314, 1-27.

21. Hayashi, M.; Floyd, J.C., Jr.; Pek, S.; Fajans, S.S. Insulin, proinsulin, glucagon and gastrin in pancreatic tumors and in plasma of patients with organic hyperinsulinism. J. Clin. Endocrinol. Metab. 1977, 44, 681-694. [CrossRef]

22. Matthews, D.R.; Hosker, J.P.; Rudenski, A.S.; Naylor, B.A.; Treacher, D.F.; Turner, R.C. Homeostasis model assessment: Insulin resistance and beta-cell function from fasting plasma glucose and insulin concentrations in man. Diabetologia 1985, 28, 412-419. [CrossRef]

23. American Diabetes, A. 2. Classification and Diagnosis of Diabetes: Standards of Medical Care in Diabetes-2020. Diabetes Care 2020, 43, S14-S31. [CrossRef] [PubMed]

24. Benjamini, Y.; Hochberg, Y. Controlling the False Discovery Rate: A practical and powerful approach to multiple testing. J. R. Stat. Soc. Ser. B Methodol. 1995, 57, 289-300. [CrossRef]

25. Xia, J.; Wishart, D.S. MSEA: A web-based tool to identify biologically meaningful patterns in quantitative metabolomic data. Nucleic Acids Res. 2010, 38, W71-W77. [CrossRef]

26. Chong, J.; Soufan, O.; Li, C.; Caraus, I.; Li, S.; Bourque, G.; Wishart, D.S.; Xia, J. MetaboAnalyst 4.0: Towards more transparent and integrative metabolomics analysis. Nucleic. Acids Res. 2018, 46, W486-W494. [CrossRef] [PubMed]

27. Ho, J.E.; Larson, M.G.; Ghorbani, A.; Cheng, S.; Chen, M.H.; Keyes, M.; Rhee, E.P.; Clish, C.B.; Vasan, R.S.; Gerszten, R.E.; et al. Metabolomic Profiles of Body Mass Index in the Framingham Heart Study Reveal Distinct Cardiometabolic Phenotypes. PLoS ONE 2016, 11, e0148361. [CrossRef]

28. de Mello, V.D.; Paananen, J.; Lindstrom, J.; Lankinen, M.A.; Shi, L.; Kuusisto, J.; Pihlajamaki, J.; Auriola, S.; Lehtonen, M.; Rolandsson, O.; et al. Indolepropionic acid and novel lipid metabolites are associated with a lower risk of type 2 diabetes in the Finnish Diabetes Prevention Study. Sci. Rep. 2017, 7, 46337. [CrossRef]

29. Heimerl, S.; Fischer, M.; Baessler, A.; Liebisch, G.; Sigruener, A.; Wallner, S.; Schmitz, G. Alterations of plasma lysophosphatidylcholine species in obesity and weight loss. PLoS ONE 2014, 9, e111348. [CrossRef] 
30. Kubota, K.; Ishizaki, T. Dose-dependent pharmacokinetics of benzoic acid following oral administration of sodium benzoate to humans. Eur. J. Clin. Pharm. 1991, 41, 363-368. [CrossRef]

31. Owen, O.E.; Reichard, G.A., Jr.; Patel, M.S.; Boden, G. Energy metabolism in feasting and fasting. Adv. Exp. Med. Biol. 1979, 111, 169-188. [CrossRef]

32. Meessen, E.C.; Bakker, G.J.; Nieuwdorp, M.; Dallinga-Thie, G.M.; Kemper, E.M.; Damink, S.W.O.; Romijn, J.A.; Hartmann, B.; Holst, J.J.; Knop, F.K.; et al. Parenteral nutrition impairs plasma bile acid and gut hormone responses to mixed meal testing in lean healthy men. Clin. Nutr. 2020, 40, 1013-1021. [CrossRef]

33. de Miranda, J.A.; Almeida, G.G.; Martins, R.I.; Cunha, M.B.; Belo, V.A.; dos Santos, J.E.; Mourao-Junior, C.A.; Lanna, C.M. The role of uric acid in the insulin resistance in children and adolescents with obesity. Rev. Paul Pediatr. 2015, 33, 431-436. [CrossRef] [PubMed]

34. Zhao, X.; Peter, A.; Fritsche, J.; Elcnerova, M.; Fritsche, A.; Haring, H.U.; Schleicher, E.D.; Xu, G.; Lehmann, R. Changes of the plasma metabolome during an oral glucose tolerance test: Is there more than glucose to look at? Am. J. Physiol. Endocrinol. Metab. 2009, 296, E384-E393. [CrossRef] [PubMed]

35. Schooneman, M.G.; Vaz, F.M.; Houten, S.M.; Soeters, M.R. Acylcarnitines: Reflecting or inflicting insulin resistance? Diabetes 2013, 62, 1-8. [CrossRef] [PubMed]

36. Kelley, D.E.; Mandarino, L.J. Fuel selection in human skeletal muscle in insulin resistance: A reexamination. Diabetes 2000, 49, 677-683. [CrossRef]

37. Dimitriadis, G.; Mitrou, P.; Lambadiari, V.; Maratou, E.; Raptis, S.A. Insulin effects in muscle and adipose tissue. Diabetes Res. Clin. Pr. 2011, 93 (Suppl. 1), S52-S59. [CrossRef]

38. Mihalik, S.J.; Michaliszyn, S.F.; de las Heras, J.; Bacha, F.; Lee, S.; Chace, D.H.; DeJesus, V.R.; Vockley, J.; Arslanian, S.A. Metabolomic profiling of fatty acid and amino acid metabolism in youth with obesity and type 2 diabetes: Evidence for enhanced mitochondrial oxidation. Diabetes Care 2012, 35, 605-611. [CrossRef] [PubMed]

39. Vangipurapu, J.; Stancakova, A.; Smith, U.; Kuusisto, J.; Laakso, M. Nine Amino Acids Are Associated With Decreased Insulin Secretion and Elevated Glucose Levels in a 7.4-Year Follow-up Study of 5,181 Finnish Men. Diabetes 2019, 68, 1353-1358. [CrossRef]

40. Angelin, B.; Bjorkhem, I.; Einarsson, K.; Ewerth, S. Hepatic uptake of bile acids in man. Fasting and postprandial concentrations of individual bile acids in portal venous and systemic blood serum. J. Clin. Investig. 1982, 70, 724-731. [CrossRef] [PubMed]

41. Liddle, R.A.; Goldfine, I.D.; Rosen, M.S.; Taplitz, R.A.; Williams, J.A. Cholecystokinin bioactivity in human plasma. Molecular forms, responses to feeding, and relationship to gallbladder contraction. J. Clin. Investig. 1985, 75, 1144-1152. [CrossRef] [PubMed]

42. Legry, V.; Francque, S.; Haas, J.T.; Verrijken, A.; Caron, S.; Chavez-Talavera, O.; Vallez, E.; Vonghia, L.; Dirinck, E.; Verhaegen, A.; et al. Bile Acid Alterations Are Associated With Insulin Resistance, but Not With NASH, in Obese Subjects. J. Clin. Endocrinol. Metab. 2017, 102, 3783-3794. [CrossRef] [PubMed]

43. Hannon, T.S.; Janosky, J.; Arslanian, S.A. Longitudinal study of physiologic insulin resistance and metabolic changes of puberty. Pediatr. Res. 2006, 60, 759-763. [CrossRef] [PubMed]

44. Ballerini, M.G.; Bergada, I.; Rodriguez, M.E.; Keselman, A.; Bengolea, V.S.; Pipman, V.; Domene, H.M.; Jasper, H.G.; Ropelato, M.G. Insulin level and insulin sensitivity indices among healthy children and adolescents. Arch. Argent. Pediatr. 2016, 114, 329-336. [CrossRef]

45. Arslanian, S.A.; Heil, B.V.; Becker, D.J.; Drash, A.L. Sexual dimorphism in insulin sensitivity in adolescents with insulin-dependent diabetes mellitus. J. Clin. Endocrinol. Metab. 1991, 72, 920-926. [CrossRef] [PubMed]

46. Iyer, G.R.; Wigginton, J.; Duren, W.; LaBarre, J.L.; Brandenburg, M.; Burant, C.; Michailidis, G.; Karnovsky, A. Application of Differential Network Enrichment Analysis for Deciphering Metabolic Alterations. Metabolites 2020, 10, 479. [CrossRef] [PubMed] 